\title{
DIVERSIDAD, ABUNDANCIA Y ESTACIONALIDAD DEL POLEN alergénico en la atmósfera de Mar del Plata (ARgentinA)
}

\author{
DIVERSITY, ABUNDANCE AND SEASONALITY OF THE ALLERGENIC AIRBORNE \\ pollen in Mar del Plata (Argentina)
}

\author{
M. Laura Abud Sierra ${ }^{1}$ y Fabiana Latorre ${ }^{2 *}$ (iD
}

1. Facultad de Ciencias Exactas y Naturales. Universidad Nacional de Mar del Plata. Av. Juan B. Justo 2550. 7600 Mar del Plata. Argentina.

2. Instituto de Investigaciones Marinas y Costeras (IIMyC), CONICET-UNMDP, FCEyN. Av. Juan B. Justo 2550. 7600 Mar del Plata. Argentina.

*fabianalatorre@yahoo.com.ar

Citar este artículo ABUD SIERRA, M. L. \& F. LATORRE. 2020. Diversidad, abundancia y estacionalidad del polen alergénico en la atmósfera de Mar del Plata (Argentina). Bol. Soc. Argent. Bot. 55: 3-21.

DOI: https://doi. org/10.31055/1851.2372.v55. n1.23094

Recibido: 14 Enero 2019

Aceptado: 11 Noviembre 2019

Publicado: 25 Marzo 2020

Editor: Gonzalo Márquez (D)

ISSN versión impresa 0373-580X

ISSN versión on-line 1851-2372

\section{SUMMARY}

Background and aims: Pollen grains represent one of the main biological pollutants in the air causing allergies in the population. To know the biological quality of the air and how it varies throughout the year, is essential to implement therapeutic and preventive measures. It was proposed: 1) to determine the allergenic potential of the atmospheric pollen spectrum of the city of Mar del Plata, 2) to elaborate a pollen calendar with the most allergenic taxa, and 3) to establish pollen stations that allow to identify the periods with greater incidence of pollen, recognizing the main allergenic contributors.

M\&M: Aerobiological monitoring was carried out for one year with a volumetric and continuous aerobiological sampler.

Results: The average daily concentrations totaled 12742 grains. Fifty pollen types were recorded; 30 were allergenic ( 21 arboreal and 9 herbaceous). The taxa with a moderate or high allergenicity were: Cupressaceae, Poaceae, Cedrus and Platanus with more than 5\% per year, and Acer, Urticaceae, Moraceae, Myrtaceae, Populus, Ulmus and Fraxinus with values between 2 and $5 \%$. Also Ambrosia and Artemisia that are highly allergenic, all were included in a pollen calendar to visualize the duration, intensity and seasonality of each individual pollination period. Five main pollen stations were defined by the abundase of taxa with high levels of allergenicity: February-March, April, August, September-October, November-December.

Conclusions: Ninety-five percent of airborne pollen grains along the year are allergenic or potentially allergenic. Aerobiological monitoring continues to incorporate interannual variations.

KEY WORDS

Aerobiology, urban environment, allergenic pollen, anual variation.

\section{RESUMEN}

Introducción y objetivos: Los granos de polen representan uno de los principales contaminantes biológicos del aire ya que causan alergias en gran parte de la población. Conocer la calidad biológica del aire y cómo varía a lo largo del año, es fundamental para implementar medidas terapéuticas y preventivas. En este trabajo, se planteó: 1) determinar la potencialidad alergénica del espectro polínico atmosférico de la ciudad de Mar del Plata, 2) elaborar un calendario polínico con los taxones más alergénicos, y 3 ) establecer estaciones polínicas que permitan identificar los periodos con mayor incidencia de polen alergénico, reconociendo los principales contribuyentes.

M\&M: Se realizó un monitoreo aerobiológico durante un año con una muestreador aerobiológico, volumétrico y continuo.

Resultados: Las concentraciones medias diarias sumaron 12742 granos. Se registraron 50 tipos polínicos, 30 de los cuales fueron alergénicos (21 arbóreos y 9 herbáceos). Los taxones con una alergenicidad moderada o alta fueron: Cupressaceae, Poaceae, Cedrus y Platanus con más del $5 \%$ anual, y Acer, Urticaceae, Moraceae, Myrtaceae, Populus, Ulmus y Fraxinus con valores entre 2 y $5 \%$. Con ellos, más Ambrosia y Artemisia que son altamente alergénicos, se estableció un calendario polínico que permitió visualizar la duración, intensidad y estacionalidad de cada período de polinación individual. Además, se determinaron cinco estaciones polínicas definidas por taxones abundantes y con niveles altos de alergenicidad: febrero-marzo, abril, agosto, setiembre-octubre, noviembre-diciembre.

Conclusiones: El 95\% del polen anual es alergénico o potencialmente alergénico. Se continúa con el monitoreo aerobiológico para incorporar las variaciones interanuales.

\section{Palabras clave}

Aerobiología, ambiente urbano, polen alergénico, variación anual. 


\section{INTRODUCCIÓN}

La atmósfera contiene un complejo grupo de partículas en suspensión, y la Aerobiología estudia la diversidad y abundancia de aquellas que tienen origen biológico como los granos de polen, su relación con el ambiente y su impacto sobre los organismos (Edmonds \& Benninhoff, 1973). Los resultados son útiles en diversas disciplinas científicas, por ejemplo en Ecología, ya que del análisis aerobiológico a largo plazo pueden extraerse conclusiones valiosas sobre las variaciones de las especies que componen un determinado ecosistema y por lo tanto como herramienta para estudios del Cambio Climático (Fernanadez-Llamazares et al., 2014); en Agronomía, porque los datos aerobiológicos son indicadores de la futura producción de cosecha en especies de elevado valor económico (Simón et al., 2018); y específicamente, en Medicina, ya que el polen es el agente causante de alergias estacionales o polinosis. Los granos de polen suspendidos en el aire ingresan a las vías respiratorias superiores durante la inspiración y las proteínas que contienen se disuelven en las mucosas e ingresan a la circulación sanguínea. El contacto repetido con estas sustancias extrañas (alérgenos), estimula el sistema inmunológico produciendo anticuerpos IgE (Huecas Gayo, 2001). Las reacciones generadas se manifiestan como rinitis, conjuntivitis y asma bronquial (Huertas Amorós et al, 2013). Cerca de 400 millones de personas en el mundo padecen rinitis alérgica (D'Amato et al., 2007); diferentes estudios epidemiológicos han demostrado que su prevalencia en los países desarrollados con un estilo de vida occidental, abarca entre el $23 \%$ y el $40 \%$ de la población europea (Millares Lopez, 2015). En Argentina según Fundaler (Salmud \& Salmud, 2016), una de cada cinco personas padece alergias, y el polen es el segundo factor causal. Actualmente, se sugiere que la contaminación del aire en las ciudades, debidas principalmente al intenso tráfico vehicular y la actividad de las industrias, aumenta la sensibilidad de las vías aéreas dañando las mucosas, lo que facilita la penetración de los alérgenos (Terán et al., 2009).

Debido a la importancia epidemiológica de la polinosis, desde mediados del siglo XX se consideró importante realizar recuentos ambientales de los tipos polínicos. El análisis aerobiológico permite establecer la dinámica atmosférica de los mismos, con lo cual es posible tratar con más exactitud las alergias respiratorias causadas por el polen (Hussain y Smith, 2003). Conocer con anticipación cuando comienza la estación en la cual la cantidad del polen en la atmósfera que causa rinitis o asma, es alto o superior al valor crítico, facilita que los pacientes sensibles tomen las medidas de precaución adecuadas.

Tanto la composición (diversidad) de tipos polínicos en el aire urbano, como la estacionalidad, intensidad y extensión de los períodos de polinación, varían a lo largo del año en función de la fenología de las especies que componen la flora urbana y de sus alrededores, y que aportan polen al espectro atmosférico durante su época reproductiva. Los parámetros de estas variables para los tipos polínicos alergénicos, son bioindicadores importantes de la calidad biológica del aire.

El carácter alergógeno según los postulados de Thommen (Mathov, 1972), se basan en que el polen debe ser activo, anemófilo, producirse en cantidad suficiente, liviano, y las plantas que lo producen deben tener una amplia distribución. La flora de muchas ciudades argentinas, entre ellas Mar del Plata, cumple con estos postulados (Latorre, 1999a). Las fuentes de polen más importantes son los árboles plantados en calles y zonas verdes de recreación, o las plantas que crecen espontáneas en terrenos abandonados o en zonas rurales o naturales circundantes.

Dentro de América del Sur, Mar del Plata es la primera ciudad con estudios aerobiológicos, los que se desarrollaron entre 1987 y 1997 (Bianchi, 1994; Latorre \& Bianchi, 1997 y 1998; Latorre \& Pérez, 1997; Latorre, 1999a; Pérez, 2000; Latorre \& Caccavari 2009). Estos trabajos se centraron en el conocimiento teórico de la productividad polínica de las plantas y del transporte eólico del polen, pero no se relacionaron con su aplicabilidad en alergias como un servicio a la población. En las últimas décadas varios países han comenzado a realizar estudios aerobiológicos directamente relacionados con alergias como la Red Española de Aerobiología (REA) (García Mozo, 2002), la Red Europea de Alergia "European Allergy Network" (EAN), y la red de la American Academy of Allergy, Asthma \& Immunology (AAAAI) que incluye a la Red de la Asociociación Argentina de Alergia e Inmunología Clínica (AAAeIC). En esta última, es que Mar del 


\section{L. Abud Sierra y F. Latorre - Polen alergénico en Mar del Plata}

Plata se inserta con un servicio STAN CONICET desde 2013 (Latorre et al., 2014; Abud et al. 2015). Como contribución al conocimiento de la calidad biológica del aire, se propone identificar a los bioindicadores para Mar del Plata, detectando cuáles son tipos polínicos alergénicos, en qué momento del año se encuentran y en qué cantidad. Ello contribuirá a realizar diagnósticos más precisos y tratamientos más específicos, incluida la inmunoterapia.

\section{Materiales y Métodos}

Características generales de la ciudad de Mar del Plata

La ciudad de Mar del Plata está ubicada a 15 metros sobre el nivel del Océano Atlántico $\left(38^{\circ} 00^{\prime} 08^{\prime \prime} \mathrm{S}, 57^{\circ} 33^{\prime} 27^{\prime \prime} \mathrm{O}\right)$, posee 39.2 $\mathrm{km}$ de costa y sus playas la hacen una de las ciudades balnearias más importantes del país (Fig. 1). La población estable aproximada es de 650.000 habitantes, y el arribo de turistas supera los 8.000.000 anuales.

El clima de Mar del Plata es templado oceánico de acuerdo a la clasificación climática de KöppenGeiger, con precipitaciones abundantes durante todo el año. La temperatura media en invierno es de $12^{\circ} \mathrm{C}$ y en verano de $23^{\circ} \mathrm{C}$; la oscilación térmica anual no es elevada. El clima de la ciudad podría definirse como subhúmedo-húmedo, mesotermal, con escasa o nula deficiencia de agua (Latorre, 1993).

La vegetación regional corresponde a una estepa de gramíneas (Provincia Pampeana). Hacia el Este de la ciudad, se presentan bosques xerófilos relictuales de tala (Provincia del Espinal). También, hay numerosas comunidades edáficas: cercanas a los cuerpos de agua dulce como juncales, totorales y pajonales de cortadera, y en la zona serrana: matorrales de curro y chilca (Cabrera, 1958; Cabrera \& Zardini, 1978). En la ciudad, los géneros de árboles plantados con alta densidad y alta frecuencia en las calles son anemófilos: Fraxinus, Ulmus, Populus, Platanus, Acer, Callistemon, Betula, Cupressus, Salix y Cedrus, excepto los entomófilos Prunus y Lagerstroemia (Latorre \& Bianchi, 1997). Las plantas herbáceas más importantes corresponden a las familias Poaceae, Asteraceae, Chenopodiaceae, Apiaceae,

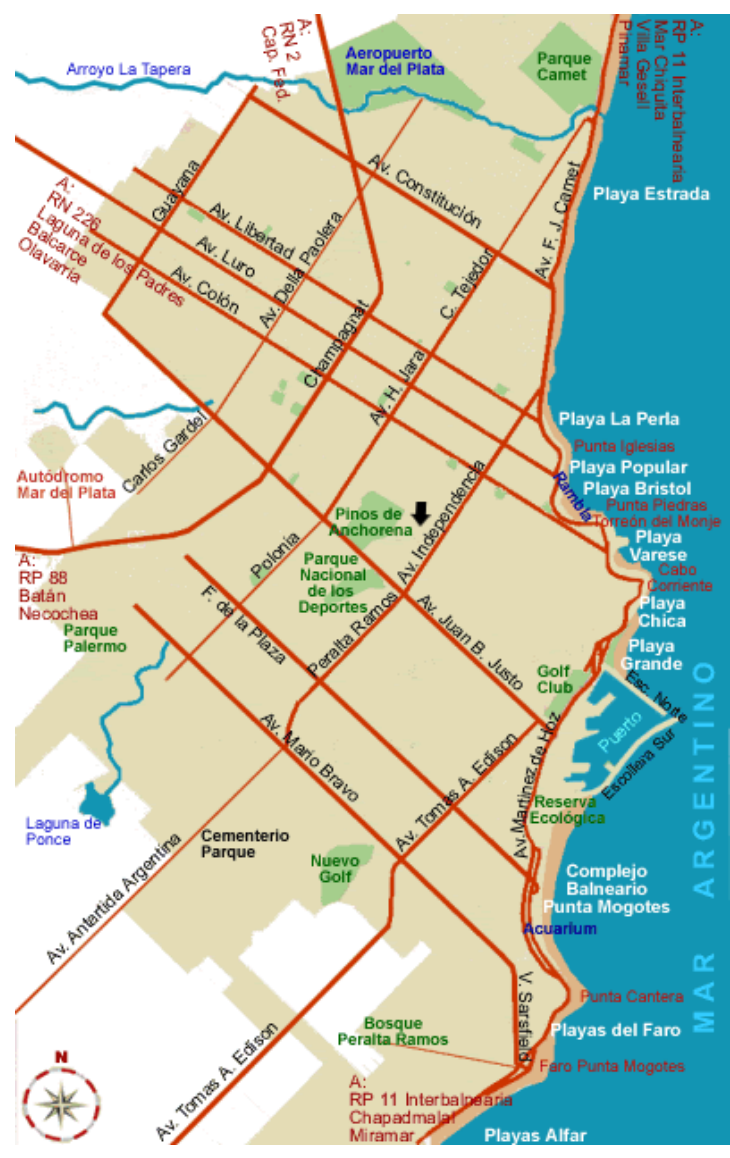

Fig. 1. Plano de la ciudad de Mar del Plata con la localización del equipo de muestreo indicada con una flecha. Fuente: sitiosargentina.com.ar/categorías/ rutas/mapas_de_rutas/calles-mar-del-plata.htm.

Brassicaceae y Polygonaceae. Dentro del paisaje urbano hay una gran variedad de micro-hábitats que contribuyen a la diversidad específica. Las especies herbáceas dominantes son introducidas, cultivadas y/o adventicias; más de la mitad corresponden a esta última categoría (Latorre, 1999a).

\section{Características del muestreo aerobiológico}

El registro polínico atmosférico de la ciudad de Mar del Plata, Argentina, se obtuvo con un muestreador aerobiológico volumétrico tipo Hirst (1952) ubicado a 15 metros de altura sobre una de las terrazas del edificio de la Facultad de Ciencias Exactas y Naturales de la Universidad Nacional de Mar del Plata (Fig. 2). Esta localización corresponde 


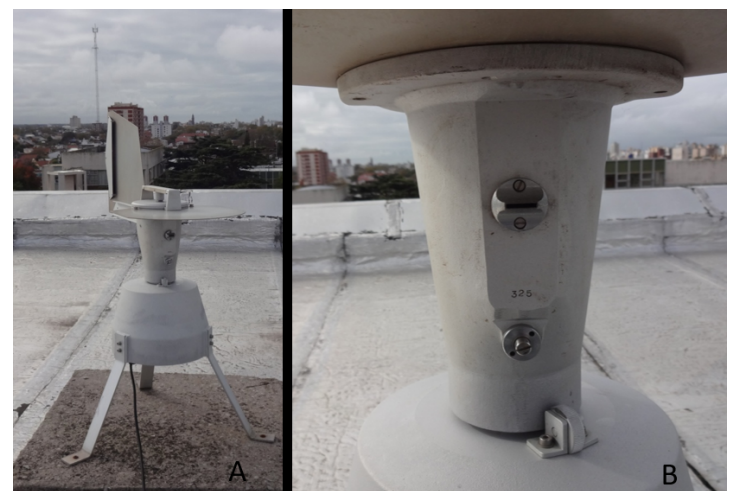

Fig. 2. Captador volumétrico y continuo tipo Hirst utilizado (A) y detalle (B).

al centro geográfico de la ciudad y es adecuada para la representación de la vegetación de la ciudad (Latorre, 1999a). Además, tiene libre circulación de vientos, sin barreras arquitectónicas ni filtros biológicos.

El muestreo aerobiológico abarcó de setiembre 2013 a agosto 2014. El periodo anual de estudio comenzó en primavera ya que en un clima templado como el de Mar del Plata, son escasas las plantas que florecen en invierno (Latorre \& Pérez, 1997).

El muestreador LANZONI VPPS 2000 utilizado, es de uso habitual en el mundo: Red Española de Aerobiología (REA) y Red Europea de Aerobiología (EAN), ya que colecta las partículas contenidas en el aire a un flujo de 10 litros por minuto (similar a la respiración humana y por ende, ideal para los estudios de alergias respiratorias). El procesamiento de las muestras se realizó según Latorre \& Caccavari (2010).

Durante el análisis al microscopio óptico para calcular los valores absolutos de cada tipo polínico, se realizaron recuentos horarios sobre transectas perpendiculares a la dirección de giro del tambor. Se analizaron 12 transectas por día, que permite una estimación insesgada de la media diaria (Käpylä y Pentinen, 1981). Para calcular la concentración media diaria de granos de polen en el aire (número de granos de polen por metro cúbico de aire: $\mathrm{p} / \mathrm{m}^{3}$ ), se siguió el protocolo de la Pan-American Aerobiology Association (Rogers \& Muilenberg, 2001). Para establecer la cantidad de polen de un período mayor al día, se sumaron las concentraciones medias diarias del mismo y se expresó como polen ${ }_{*}$ día $_{*} \mathrm{~m}^{-3}$ (Galán et al., 2017). Se utilizó un microscopio Leica DM500.

La identificación de los tipos polínicos se realizó consultando bibliografía específica (Erdtman, 1971; Heusser, 1971; Markgraf \& D’Antoni, 1978; Moore \& Webb, 1978; Moore et al., 1991; Roubik \& Moreno, 1991; Pire et al., 1998; 2001; entre otros) y material de referencia confeccionado con la técnica Wodehouse (1965) y conservado en el IIMyC (CONICET-UNMDP). En los casos en que se determinó a nivel de especie, se debió a la comparación confirmativa con los preparados de referencia realizados con la técnica Woodehouse (Erdtman, 1954). Los granos de polen que no pudieron ser determinados, los deteriorados o con características morfológicas visibles insuficientes como para ser identificados, representaron menos del $2 \%$ y no fueron incluidos en los análisis. Si bien los tipos Ambrosia y Artemisia corresponden al taxón Asteraceae Asteroideae, se los consideró aparte por su morfología distinguible y su tipo de polinización anemófila (Murray, 2006), además de su capacidad altamente alergizante (Valero \& Cahia, 2002). En el caso del tipo polínico Papilionaceae se lo considera principalmente arbóreo ya que el registro de estos granos coinciden con la floración de árboles comunes presentes en las calles de la ciudad como Robinia pseudoacacia y Stiphnolobium japonicum, según observaciones personales.

\section{Análisis de la información}

Para determinar si el periodo de estudio podía considerarse como un año típico en cuanto a sus condiciones meteorológicas, se realizó un análisis comparativo de temperaturas, precipitaciones y humedad relativa con los promedios históricos de la ciudad de Mar del Plata (Servicio Meteorológico Nacional, Estación Meteorológica Mar del Plata Aeropuerto).

Se estableció el período de polinación (PP) de cada taxón, el cual se extiende desde el día en que se registra el primer grano de polen hasta el día en que se registra el último. Debido a que ciertos tipos polínicos son muy livianos y pueden resuspenderse por fuertes vientos, se considera que un grano de polen no está dentro del PP si es precedido o es seguido por más de 10 días sin el registro de ningún grano del mismo tipo. También se determinó el período de polinación principal (PPP), que 


\section{L. Abud Sierra y F. Latorre - Polen alergénico en Mar del Plata}

elimina los días en los cuales el nivel de polen es mínimo: los primeros y últimos días del PP que no corresponden generalmente a la floración de la vegetación local. Para definirlo, se utilizó el criterio de Nilsson \& Persson (1981) que lo considera PPP desde el día en que la suma de las concentraciones medias diarias alcanzan el 5\% del total de polen producido por el taxón en el año, hasta el día en que la suma llega al $95 \%$.

Se calcularon los valores de las fases más importantes de cada curva polínica individual. Los parámetros que definen la estacionalidad son: día de inicio y final del PP y PPP, duración en días del PP y PPP, día en que se registra el máximo de concentración; y los que definen la intensidad son: total anual y concentración máxima alcanzada.

La alergenicidad de los tipos polínicos se determinó utilizando los listados de la Unión Internacional de Sociedades de Inmunología (2019), el Manual de Polinosis de Valero \& Cahia (2002) y trabajos científicos donde también se prueba la presencia de sustancias antigénicas o el efecto en la población. Entre ellos, Bousquet et al. (1993), Ardusso et al. (2010) y Marchuet (2016) donde adjudican alergenicidad a diversas especies de Cupressaceae; Van Ree et al. (1998) y De la Plaza Aguado (2018) mencionan diferentes especies de Poaceae con reactividad cruzada y Jato et al. (2000) atribuye umbrales de reacción; Ibarrola et al. (2004) detecta alérgenos específicos en Platanus acerifolia; alergenicidad de Ulmus, Salix, Populus, Pinus, Plantago, Myrtaceae con especies del género Eucalyptus, Morus spp., Juglans regia, Castanea sativa, Betula pendula, especies de la familia Asteraceae y ChenopodiaceaeAmaranthaceae, Acer (Giner, 1999); Platanus y Urticaceae (Castro Alfageme, 2015), Ambrosia y Artemisia (Puiggròs Casas, 2012), Ligustrum (Cariñanos et al., 2002); Ginkgo biloba (Kochibe, 1997); las especies de Fraxinus ya en Pla Dalmau (1961) son consideradas alergénicas; Casuarina (Cid Martinez et al., 2013); Celtis tala (Baroni et al, 2008); Cedrus y Liquidambar styraciflua (Valero \& Cahia, 2002); Oleaceae (Ferreira et al., 2014) y Ricinus communis (Garcia-Gonzalez et al., 1999).

Se elaboró un calendario polínico para conocer el espectro polínico de la localidad a lo largo del año, que permitirá reconocer a los principales tipos de polen con potencialidad alergénica que los médicos y pacientes afectados de polinosis deben tener en cuenta. El calendario polínico es una representación gráfica que comprende en una sola figura la dinámica anual de los principales tipos polínicos de una localidad, ordenados en función de su periodo de polinación y destacando la importancia relativa de cada uno de ellos. Para la elaboración del calendario polínico de la ciudad de Mar del Plata se utilizaron las medias mensuales de los registros diarios. Solo se consideraron para su elaboración aquellas especies que representaron más del $2 \%$ del total anual, o bien aquellas que alcanzando más del $1 \%$, eran muy alergénicas.

Utilizando los tipos polínicos del calendario, se realizó un análisis de agrupamiento Cluster Analysis en el cual los grupos a determinar son desconocidos a priori; los elementos (meses) dentro de cada conglomerado presentan cierto grado de homogeneidad en base a los valores adoptados sobre un conjunto de variables (taxones polínicos alergénicos). En este caso, se buscó determinar estaciones de polen alergénico y no estaciones calendario; se utilizó distancia euclidiana y ligamiento simple (programa STATISTICA).

\section{Resultados}

Condiciones meteorológicas durante el periodo de estudio

Entre setiembre 2013 y agosto 2014 la temperatura media anual fue de $14^{\circ} \mathrm{C}$ y la humedad relativa promedio $79 \%$, ambos valores similares a los promedio de los últimos 10 años. En cambio, las precipitaciones sumaron $1281.6 \mathrm{~mm}, 341 \mathrm{~mm}$ más altas que los datos históricos especialmente acumulados entre fines de invierno y principios de primavera.

Diciembre y enero registraron las temperaturas más altas, mientras que junio y julio las más bajas. En agosto se observó la mayor cantidad de lluvia, seguido por setiembre, enero (concentrada en sólo tres días) y marzo; diciembre fue el menos lluvioso. La humedad relativa de mayo y julio fue alta $(85 \%$ en ambos meses) y la de enero, mínima (70\%) (Fig. 3).

\section{Caracterización del espectro polínico}

La suma anual de las concentraciones medias diarias del polen total registrado en el aire de Mar del Plata durante el periodo estudiado fue de 12742 polen ${ }_{*}$ día $_{*} \mathrm{~m}^{-3}$. El espectro atmosférico incluyó 50 

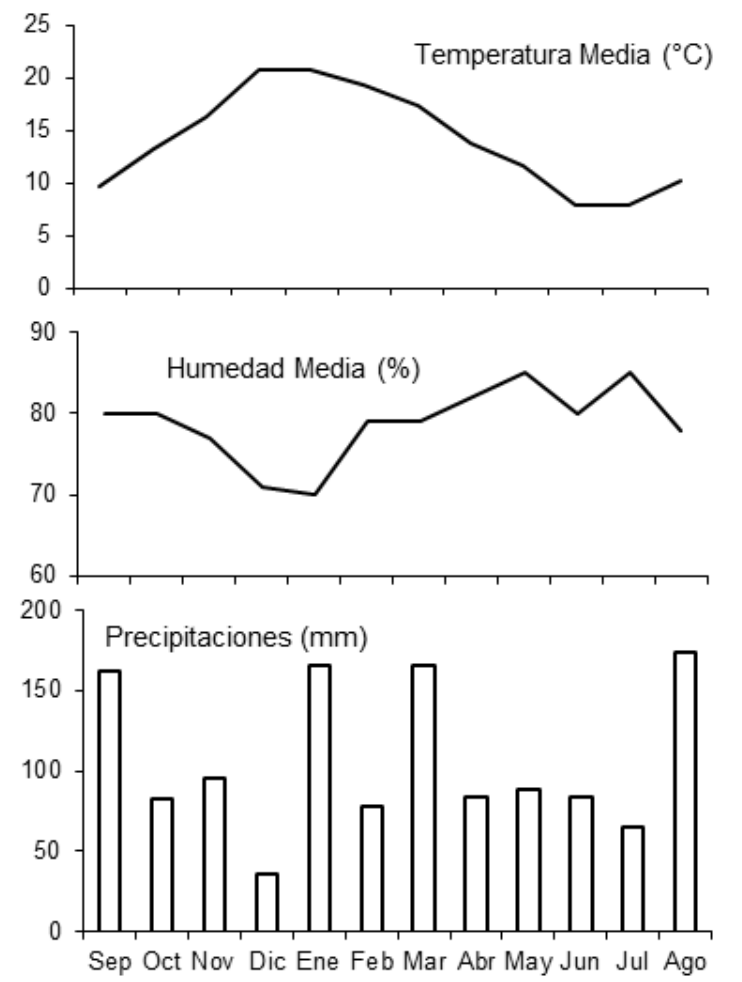

Fig. 3. Temperatura media, humedad relativa media y precipitaciones totales para Mar del Plata (setiembre 2013-agosto 2014).

tipos polínicos diferentes; 30 arbóreos $(65.8 \%$ del polen total) y 20 herbáceos. Según la bibliografía específica citada, 30 tienen capacidad alergénica: 21 arbóreos y 9 herbáceos, los que aportaron 95\% del polen total (Tabla 1). Las especies presentes en la ciudad se encuentran detalladas en Latorre (1997), Latorre (1999b), Latorre \& Bianchi (1997), Latorre et al. (2001), Latorre et al. (2008), y confirmadas por los relevamientos realizados.

Agosto fue el mes con más granos de polen en el aire $(3192,56)$ y junio el de menor concentración $(121,52)$ (Fig. 4).

En la Figura 5 se detallan los porcentajes de los tipos polínicos más abundantes registrados en cada mes del año. El predominio (valores cercanos o mayores al $50 \%$ del polen mensual) de un solo tipo polínico ocurrió en siete meses del año: Poaceae en noviembre, diciembre y enero, Cedrus en abril y mayo, y Cupressaceae en julio y agosto. En el resto de los meses (setiembre-octubre, febrero-marzo, y junio), se observaron entre cinco y siete taxones importantes.

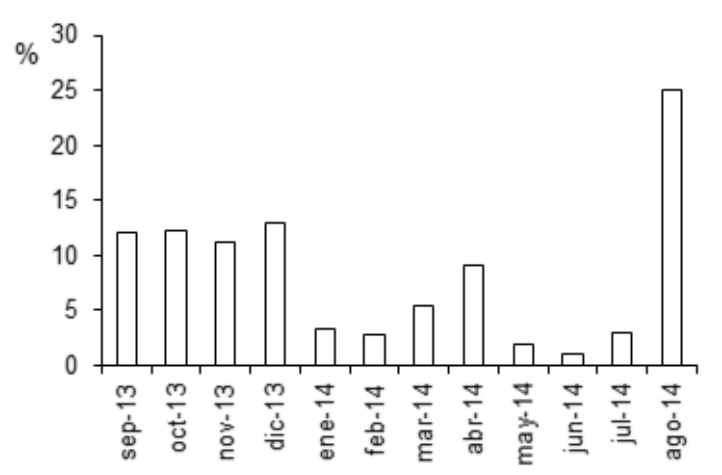

Fig. 4. Porcentaje mensual de polen en la atmósfera de la ciudad de Mar del Plata.

En primavera se registró la mayor cantidad de granos de polen (4446) representando el 35,5\% del espectro, y la menor cantidad de granos (2064) durante el otoño, 16,4\% del total anual. El polen de hierbas sólo predominó en verano y fue muy escaso en invierno (Fig. 6).

A lo largo del año, sólo 4 tipos polínicos tuvieron porcentajes mayores al 5\%, 7 con proporciones entre $5 \%$ y $2 \%$, mientras que con porcentajes del $1 \%$ se encontraron 11 tipos polínicos. El resto de los taxones no superaron el $1 \%$ de la concentración anual (Tabla 2).

El $51 \%$ del polen anual correspondió solamente a dos taxones: Cupressaceae y Poaceae, que estuvieron presentes en todos los meses del año. Los taxones arbóreos más representativos en el espectro fueron: Cedrus y Cupressaceae; Cedrus registró su máxima concentración durante el otoño ( $83 \%$ del polen registrado en abril) y Cupressaceae durante el invierno (77\% en agosto). De los taxones herbáceos, Poaceae fue el más importante y dominó entre fines de primavera y principios de verano (acumulando el 30\% de su total anual en noviembre y el $41 \%$ en diciembre).

Todos los tipos polínicos con más de un $2 \%$ fueron potencialmente alergénicos y de los que acumularon 1\%, sólo tres (Cyperaceae, Casuarina y Apiaceae) no están identificados como alergénicos.

Curvas aerobiológicas de los tipos polínicos abundantes y alergénicos

Para los cuatro tipos polínicos más abundantes y para Artemisia y Ambrosia, incluidos por 
Tabla 1. Diversidad de tipos polínicos en la atmósfera de Mar del Plata a lo largo del año. Se indica el hábito de las plantas, el tipo de polinización, si el polen es alergénico.

\begin{tabular}{|c|c|c|c|}
\hline Tipo polínico & Arbóreo/Herbáceo & Alergénico & Anemófilo/Entomófilo \\
\hline Acer & A & + & $\mathrm{A}$ \\
\hline Ambrosia & $\mathrm{H}$ & + & A \\
\hline Apiaceae & $\mathrm{H}$ & & E \\
\hline Araucaria & A & & A \\
\hline Artemisia & $\mathrm{H}$ & + & A \\
\hline Asteraceae Asteraoidea & $\mathrm{H}$ & + & E/A \\
\hline Asteraceae Cichorioideae & $\mathrm{H}$ & + & E/A \\
\hline Betula pendula & A & + & A \\
\hline Brassicaceae & $\mathrm{H}$ & & E \\
\hline Castanea sativa & A & + & A \\
\hline Casuarina & A & & A \\
\hline Cedrus & A & + & A \\
\hline Celtis & A & + & A \\
\hline Chenopodiineae & $\mathrm{H}$ & + & A \\
\hline Cupressaceae & A & + & A \\
\hline Cyperaceae & $\mathrm{H}$ & & A \\
\hline Echium plantagineum & $\mathrm{H}$ & & E \\
\hline Ephedra & $\mathrm{H}$ & & A \\
\hline Fabaceae Mimosaceae & A & & E \\
\hline Fabaceae Papilionoideae & A & & $\mathrm{E}$ \\
\hline Fraxinus & A & + & A \\
\hline Ginkgo biloba & A & + & A \\
\hline Gomphrena & $\mathrm{H}$ & & E \\
\hline Juglans regia & A & + & A \\
\hline Juncaceae & $\mathrm{H}$ & & A \\
\hline Lauraceae & A & & $\mathrm{E}$ \\
\hline Ligustrum & A & + & $A / E$ \\
\hline Liquidambar styraciflua & A & + & A \\
\hline Mnonocotyledoneae & A & & \\
\hline Morus & A & + & A \\
\hline Myoporium laetum & A & & $\mathrm{E}$ \\
\hline Myrtaceae & A & + & $A / E$ \\
\hline Nothofagus & A & & A \\
\hline Olea & A & + & $A / E$ \\
\hline Phytolacca dioica & A & & $\mathrm{E}$ \\
\hline Pinus & A & + & A \\
\hline Plantago & $\mathrm{H}$ & + & A \\
\hline Platanus acerifolia & A & + & A \\
\hline Poaceae & $\mathrm{H}$ & + & A \\
\hline Populus & A & + & A \\
\hline Quercus & A & + & A \\
\hline Rannunculaceae & $\mathrm{H}$ & & E \\
\hline Ricinus comunis & $\mathrm{H}$ & + & E \\
\hline Salix & A & + & A \\
\hline Schinus & A & & E \\
\hline Solanum & $\mathrm{H}$ & & E \\
\hline Typha angustifolia & $\mathrm{H}$ & & A \\
\hline Typha latifolia & $\mathrm{H}$ & & A \\
\hline Ulmus & A & + & A \\
\hline Urticaceae & $\mathrm{H}$ & + & A \\
\hline
\end{tabular}



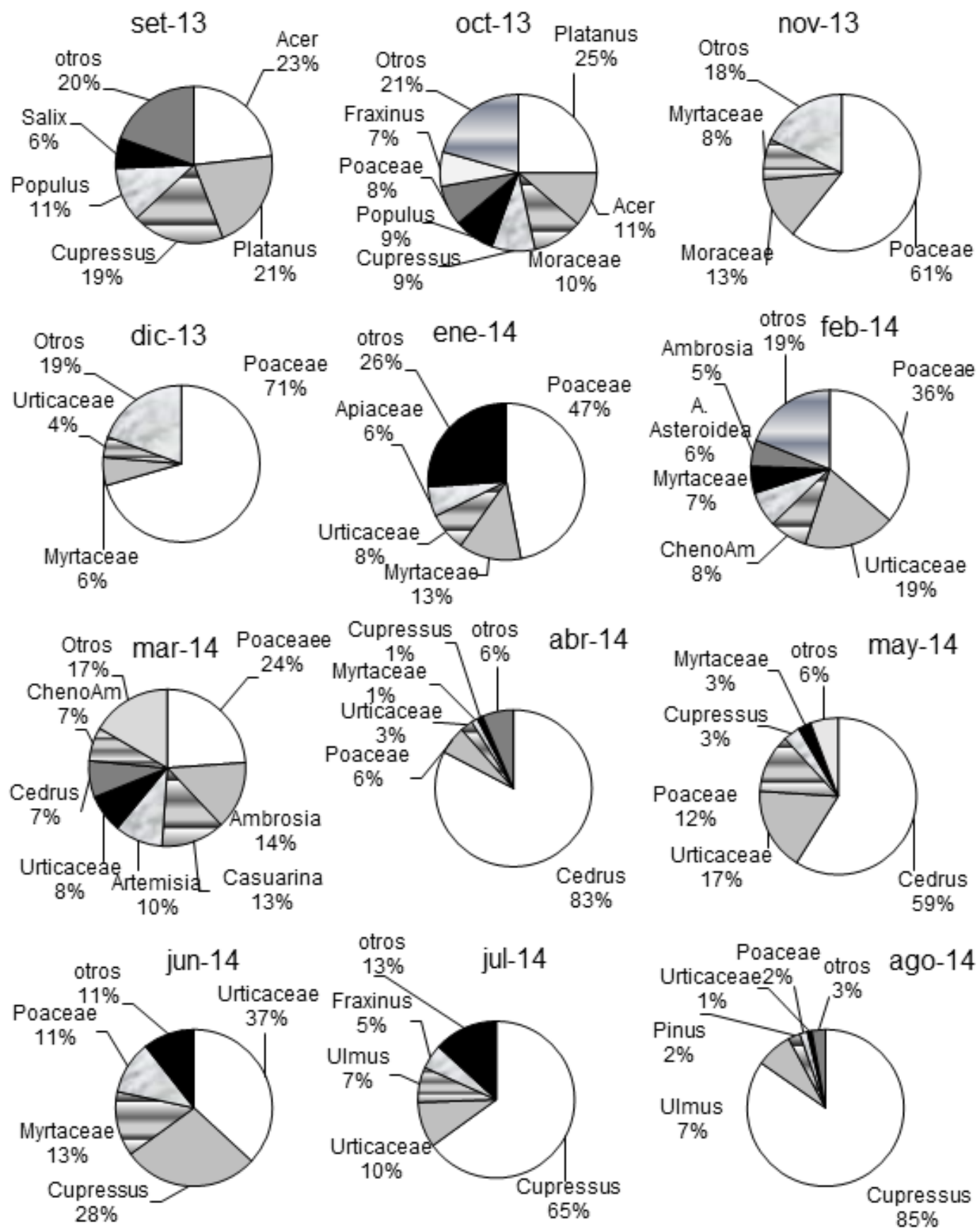

Fig. 5. Porcentaje mensual de los distintos taxones en la atmósfera de la ciudad de Mar del Plata durante el periodo de estudio.

su gran potencial alergénico, se calcularon los valores de las fases más importantes de las curvas aerobiológicas en cuanto a estacionalidad (inicio, final y duración del PP y PPP; día del máximo) e intensidad o abundancia (total anual y concentración diaria máxima) (Tabla 3 ).

Cupressaceae: este taxón es de polinización invernal, y por lo tanto al inicio del monitoreo 


\section{L. Abud Sierra y F. Latorre - Polen alergénico en Mar del Plata}

aerobiológico en setiembre, ya había comenzado su estación polínica y se registraba polen. Si consideramos ambos períodos anuales parciales, podemos estimar que el PP tiene una duración de medio año mientras que el PPP sólo abarca dos meses, acumulando un $89 \%$ del total incluyendo el día de concentración máxima en agosto. En los meses de enero, febrero, marzo y abril, no se detectó ningún grano de polen de este tipo.

Poaceae: el polen de gramíneas se observó durante todo el año; no hubo más de dos días seguidos sin la presencia de este tipo polínico en el aire, excepto durante cinco días de junio. Por convención, puede establecerse que el PP se identifica con el año calendario. Sin embargo, el PPP estuvo definido desde fines de octubre hasta principios de abril, con una extensión de cinco

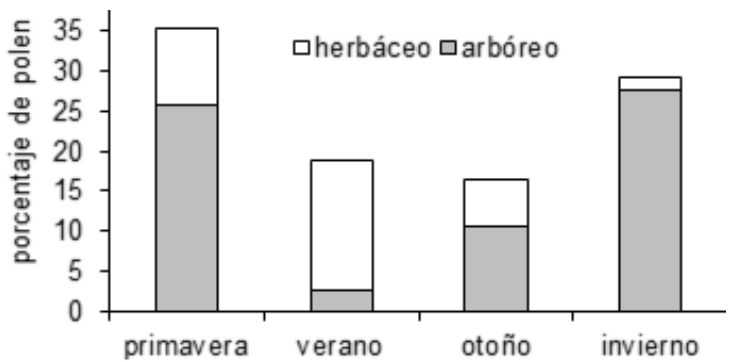

Fig. 6. Porcentaje de granos de polen de especies arbóreas y no arbóreas por estación.

meses y medio; el día de máxima concentración fue en noviembre alcanzando el $6 \%$ del total anual.

Cedrus: El PP abarcó dos meses y medio entre marzo y mayo; el PPP fue en abril con un pico de

Tabla 2. Tipos polínicos más abundantes durante el periodo agosto 2013-setiembre 2014

\begin{tabular}{|c|c|c|c|}
\hline Tipo polínico & $\begin{array}{c}\text { Abundancia } \\
\text { absoluta (p/m3) }\end{array}$ & $\begin{array}{c}\text { Abundancia } \\
\text { relativa (\%) }\end{array}$ & Escala de \% \\
\hline Cupressaceae & 3492 & 28 & \multirow{4}{*}{ más del $5 \%$} \\
\hline Poaceae & 2880 & 23 & \\
\hline Cedrus & 1155 & 9 & \\
\hline Platanus & 736 & 6 & \\
\hline Acer & 535 & 4 & \multirow{7}{*}{ entre $5 \%$ y $2 \%$} \\
\hline Urticaceae & 450 & 4 & \\
\hline Moraceae & 430 & 3 & \\
\hline Myrtaceae & 387 & 3 & \\
\hline Populus & 319 & 3 & \\
\hline Ulmus & 297 & 2 & \\
\hline Fraxinus & 197 & 2 & \\
\hline Chenopodiineae & 171 & 1 & \multirow{11}{*}{$1 \%$} \\
\hline Asteraceae Asteroidea & 134 & 1 & \\
\hline Pinus & 132 & 1 & \\
\hline Ambrosia & 128 & 1 & \\
\hline Salix & 125 & 1 & \\
\hline Celtis & 107 & 1 & \\
\hline Cyperaceae & 104 & 1 & \\
\hline Casuarina & 95 & 1 & \\
\hline Apiaceae & 88 & 1 & \\
\hline Artemisia & 69 & 1 & \\
\hline Fabaceae Papilionoideae & 64 & 1 & \\
\hline 28 tipos polínicos & 484 & 4 & menos $1 \%$ \\
\hline
\end{tabular}


Tabla 3. Fases de las curvas aerobiológicas de los taxones más abundantes y alergénicos.

\begin{tabular}{|c|c|c|c|c|c|c|}
\hline & Cupressaceae & Poaceae & Cedrus & Platanus & Ambrosia & Artemisia \\
\hline Inicio PP & 13-May & 1-Ene & 8-Mar & 8-Sep & 2-Feb & 5-Mar \\
\hline Final PP & 21-Dic & 31-Dic & 24-May & 12-Dic & 4-May & $1-A b r$ \\
\hline duración PP (días) & 222 & 365 & 78 & 96 & 94 & 29 \\
\hline inicio PPP & 21-Jul & 26-Oct & 7-Abr & 10-Sep & 23-Feb & 6-Mar \\
\hline final PPP & 25-Sep & 8-Abr & 2-May & 24-Oct & 4-Apr & 24-Mar \\
\hline duración PPP (días) & 66 & 165 & 27 & 45 & 41 & 26 \\
\hline suma polen PPP & 3118 & 2601 & 1062 & 666 & 118 & 67 \\
\hline día del máximo & 8-Ago & 23-Nov & 26-Abr & $21 / 9$ y $25 / 9$ & 23-Feb & 17-Mar \\
\hline máximo $\left(\mathrm{p} / \mathrm{m}^{3}\right)$ & 372 & 170 & 260 & 48 & 13 & 12 \\
\hline
\end{tabular}

concentración diaria que representó el $23 \%$ del total anual.

Platanus: El registro de granos comenzó a principios de setiembre y continuó hasta mediados de diciembre. El PPP fue acotado a un mes y medio (segunda quincena de setiembre y octubre), y en dos días de setiembre se observó la máxima concentración que representó el 5\% del total anual, cada uno de ellos.

Ambrosia: El PP se extendió durante tres meses (febrero, marzo y abril); el PPP sólo durante poco más de un mes, principalmente durante marzo. El día de máxima concentración se alcanzó en febrero con el $10 \%$ de la concentración total.

Artemisia: los granos de polen en la atmósfera se registraron durante marzo y el PPP comprendió menos de un mes. El 18\% del total anual se acumuló en el día de máxima concentración, ocurrido a mediados del mes.

\section{Calendario polínico}

En el calendario polínico de la ciudad de Mar del Plata, se incluyeron los tipos polínicos que alcanzaron al menos $2 \%$ del total anual, o $1 \%$ y son considerados alergénicos (Fig. 7).

En la Figura 8, se muestra la variación diaria de los cuatro tipos polínicos más abundantes en la atmósfera y se incluyó Ambrosia y Artemisia por su alta capacidad alergizante.

Algunos tipos polínicos presentaron periodos largos de polinación debido a la presencia de varias especies en la vegetación que componen cada uno de estos taxones. Por ejemplo en Poaceae: Bromus unioloides, Lolium multiflorum, Hordeum murinum, Stipa pappossa, Cynodon dactylon; en Cupressaceae: Cupressus sempervirens, C. arizonica, Juniperus communis, Thuja orientalis, en Chenopodiineae (Amaranthaceae/Chenopodiaceae): Amaranthus quitensis, Chenopodium ambrosioides, Beta vulgaris, Chenopodium muralis, en Urticaceae: Urtica urens, Urtica dioica, Parietaria officinalis, y en Myrtaceae: Eucalyptus globolus, E. camaldulensis y Callistemon lanceolatus.

Los tipos polínicos arbóreos se registraron principalmente en invierno y primavera, excepto Cedrus que dominó en otoño. Las concentraciones máximas alcanzadas por los tipos polínicos Cupressaceae y Cedrus fueron considerablemente superiores a las del resto de los taxones. Si bien Cupressaceae se registró en cantidades discretas a lo largo de todo el año, tuvo un pico durante agosto, al igual que Ulmus, aunque en menor concentración. El pico de Cedrus fue en abril. Platanus se registró durante la primavera y su pico se observó en setiembre, al igual que los máximos de Salix, Acer y Populus. Otras especies arbóreas presentaron una concentración máxima durante octubre como Fraxinus, o en noviembre: Moraceae, Myrtaceae y Celtis.

Los tipos polínicos no arbóreos presentaron períodos con mayor cantidad de granos en la atmósfera durante el verano y otoño. Sin embargo, el polen de Poaceae se registró a lo largo de todo el año con un máximo marcado en diciembre. Otras plantas herbáceas como las correspondientes al polen de Ambrosia, Artemisia, Chenopodiaceae y Amaranthaceae, alcanzaron sus máximos polínicos en marzo. 
M. L. Abud Sierra y F. Latorre - Polen alergénico en Mar del Plata
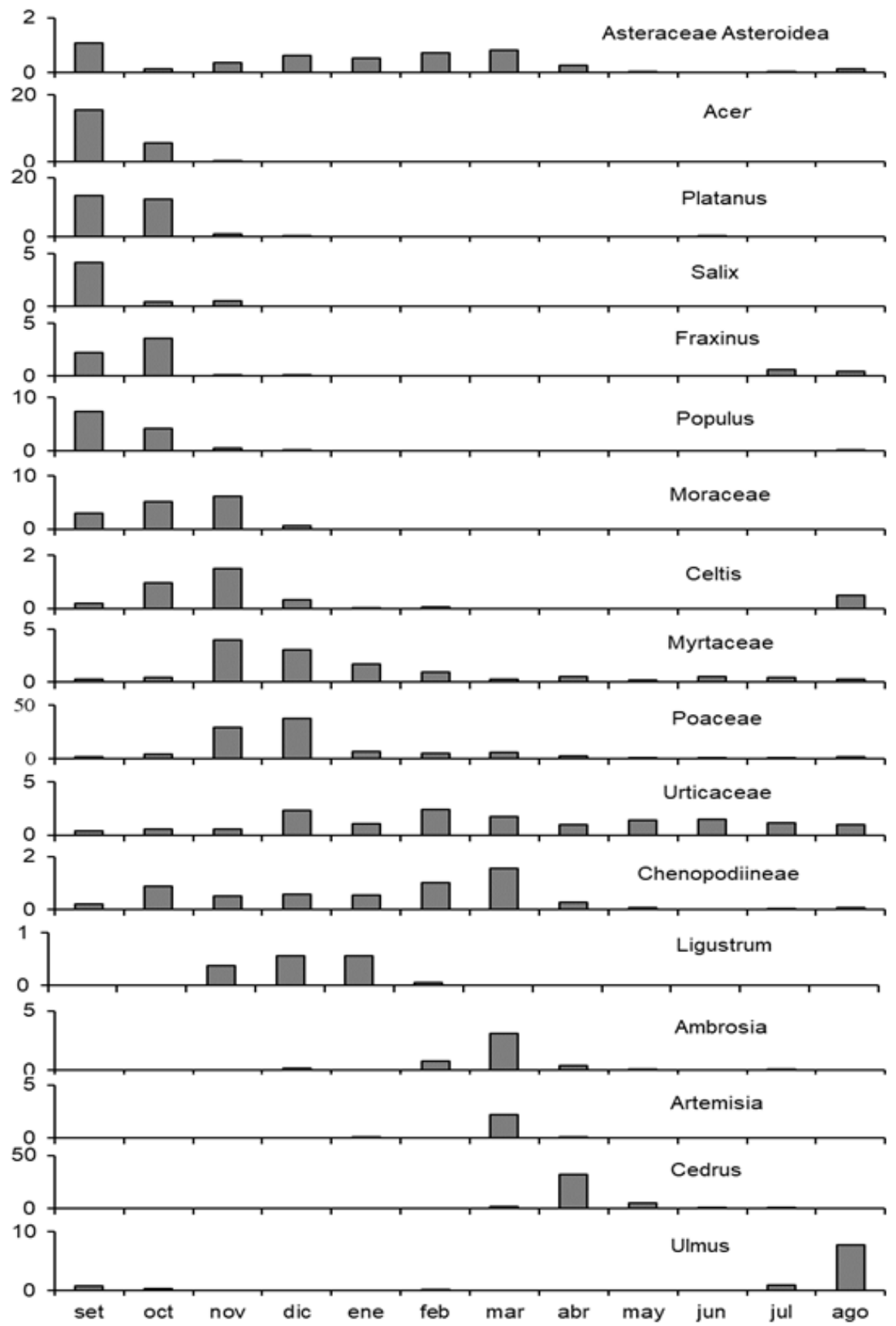

Fig. 7. Calendario polínico de las especies alergénicas más abundantes durante el periodo agosto 2013 setiembre 2014 en la ciudad de Mar del Plata.

Estaciones de polen alergénico

Se realizó un análisis de agrupamiento que permitió determinar estaciones polínicas utilizando los taxones alergénicos más abundantes: Cedrus, Celtis, Chenopodiineae, Cupressaceae, Cyperaceae,
Fraxinus, Ligustrum, Moraceae, Myrtaceae, Pinus, Platanus, Poaceae, Populus, Salix, Ulmus, Urticaceae, Acer, Ambrosia, Artemisia, A. Asteroidea. Luego del análisis de agrupamiento, el año quedó dividido en cinco grupos a una distancia de 400 (Fig. 9). 
Bol. Soc. Argent. Bot. 55 (1) 2020
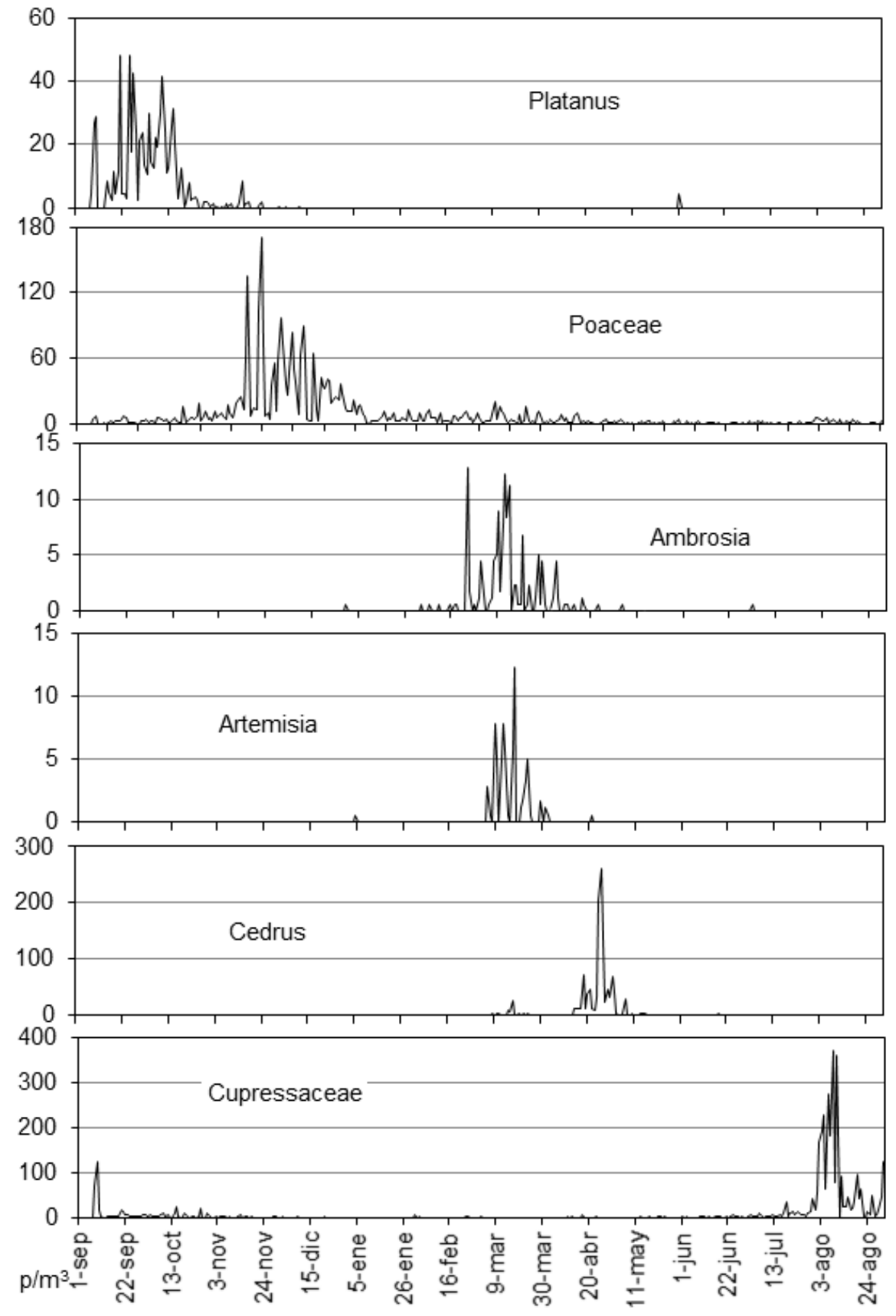

Fig. 8. Concentraciones diarias de los tipos polínicos más abundantes a lo largo del año.

El grupo 1 quedó integrado por las especies dominantes durante agosto, las más abundantes fueron las del género Cupressus. El grupo 2 se formó con las especies que dominaron durante abril e incluyó el pico de abundancia de Cedrus. El grupo
3 incluyó a las especies no arbóreas que dominaron en noviembre y diciembre, siendo el tipo polínico más abundante el de Poaceae. El grupo 4 incluyó varios meses y fue heterogéneo; marzo y junio mostraron una clara dominancia de las especies no 


\section{L. Abud Sierra y F. Latorre - Polen alergénico en Mar del Plata}

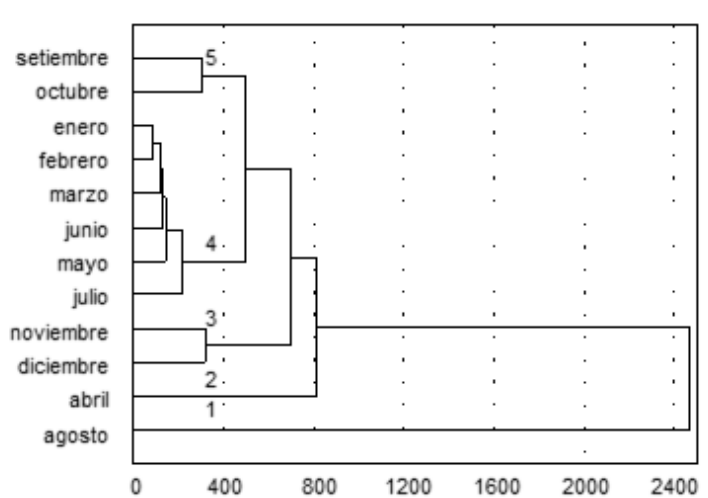

Fig. 9: Análisis de agrupamiento con los tipos polínicos alergénicos más abundantes durante el año en la ciudad de Mar del Plata.

arbóreas, mientras que mayo y julio dominancia de especies arbóreas pero también presentaron polen de especies no arbóreas como Poaceae. El grupo 5 incluyó a los meses de septiembre y octubre que estuvieron dominados por altas concentraciones de Acer y Platanus.

\section{Discusıón}

Condiciones meteorológicas durante el período de estudio

Durante el año de estudio del espectro polínico de la ciudad no se registraron eventos meteorológicos particulares. El periodo analizado no fue diferente respecto de los promedios históricos de la ciudad en cuanto a temperatura y humedad; sólo las precipitaciones mostraron valores algo mayores.

\section{Caracterización del espectro polínico}

En el aire de la ciudad de Mar del Plata, hay un número relativamente bajo de tipos polínicos importantes, como Cupressaceae y Poaceae que suman la mitad del polen anual. Sin embargo, el espectro polínico desde setiembre 2013 a agosto 2014, mostró un alto nivel de diversidad. El número de taxones hallados en el aire de la ciudad (50) es similar al descripto en estudios anteriores (Bianchi, 1994; Latorre, 1999a). Es más diverso que el descripto por Nitiu (2009) para la ciudad de La Plata donde se identifican 24 taxones y que el espectro de la ciudad de Jujuy descripto por Torres \& Flores (2013) donde se registran 36 tipos polínicos. Sin embargo, es menor que el descripto por Murray (2006) para la ciudad de Bahía Blanca (64 tipos polínicos) y por Latorre \& Caccavari (2010) (68 tipos polínicos) para la ciudad de Diamante. Cabe aclarar que el trabajo de Murray (2006) se realizó analizando periodos mayores, por lo que incluye a taxones esporádicos. Una característica en común que muestran todos los estudios, incluido éste, es la dominancia de las especies exóticas por sobre las nativas en los espectros polínicos urbanos. En general, se encontraron las mismas especies descriptas por Bianchi (1992), por Latorre \& Pérez (1997) y por Latorre \& Caccavari (2009) pero no polen de especies como Chorisia speciosa, Viburnun tinus,y de los géneros Sapium, Rumex, Bougainvillea. Los cambios observados podrían deberse a una renovación del arbolado y un cambio en la utilización de especies entomófilas en los jardines y parques de la ciudad.

Por otra parte, el espectro anual estuvo dominado por especies arbóreas apoyando lo observado en años anteriores para el mismo sitio por Bianchi (1994), Latorre \& Pérez (1997), Latorre (1999a) y Pérez (2000). Estos resultados también coinciden, con los de otras ciudades como Buenos Aires (Nitiu et al., 2003), La Plata (Nitiu, 2006), Bahía Blanca (Murray, 2006), Montevideo (Uruguay) (Tejera \& Beri, 2005). Las leves diferencias podrían deberse a condiciones climático-meteorológicas y a la composición de la vegetación urbana. El principal aporte en Mar del Plata, fue de especies exóticas con polinización anemófila de uso ornamental o de uso frecuente tanto para el arbolado de calles o espacios públicos (plazas, parques, áreas verdes). Sólo en verano se observó predominio del polen de hierbas.

La concentración polínica total presentó un pico en agosto, descendiendo hacia los meses invernales con un mínimo en junio. El máximo de agosto se debe al aporte de Cupressaceae, cuyo principal componente es Cupressus, género más abundante en la vegetación. La copiosa productividad de las especies de esta familia y los rasgos aerodinámicos de sus granos de polen favorables para el mantenimiento en suspensión, explican los altos valores de concentración atmosférica (Latorre, 1999b). El periodo septiembre-octubre estuvo dominado por especies arbóreas como Cupressaceae, Acer y Platanus. En los meses de noviembre a marzo las especies no arbóreas fueron 
predominantes, con Poaceae como principal taxón, seguido en importancia por Ambrosia, Artemisia y Urticaceae. El resto de los meses, a excepción de abril que presentó un máximo en la concentración de Cedrus, son heterogéneos en cuanto a su composición en especies arbóreas y no arbóreas.

Curvas aerobiológicas de los tipos polínicos abundantes y alergénicos

Se analizaron las curvas aerobiológicas de los tipos polínicos con más del $5 \%$ anual (Cupressaceae, Poaceae, Cedrus y Platanus), incluyendo a Ambrosia y a Artemisia por su alto potencial de alergenicidad. La duración relativa entre PP y PPP fue diferente según los taxones. Para Cupressaceae y Poaceae, el aporte de varias especies dentro de la familia con diferentes momentos de polinización hace que estos períodos sean largos y la diferencia entre ellos no sea importante. En la ciudad de Bahía Blanca ocurre algo similar: Cupressaceae se registra todo el año y Poaceae durante 10 meses (Murray et al., 2002). Estos dos taxones dominaron el espectro de Mar del Plata en décadas previas (Bianchi, 1992; Latorre \& Pérez, 1997) y son los más importantes en Diamante (Latorre \& Caccavari, 2010) y en Tucumán (García, 2010). Para Cupressaceae también es posible la re-incorporación de los granos ya depositados a la atmósfera debido al bajo peso de los mismos. Específicamente para Poaceae, estos resultados coinciden con lo descripto por de Castro Alfageme (2015) para la ciudad de Valladolid (España). Los PPP de Cedrus, Platanus, Ambrosia y Artemisia se ubican dentro de una estación calendario específica. Entre fines de verano y principios de otoño dominan las malezas, como Ambrosia y Artemisia, con relativamente baja abundancia pero alta alergenicidad. Resultados similares fueron encontrados por Marcó et al. (2014) en Concepción del Uruguay: niveles bajos de polen de Artemisia y Ambrosia pero importantes como causa de polinosis en la población de esa ciudad. El polen de Cedrus caracteriza al otoño con muy breve PP, básicamente atribuido a $C$. deodara . Finalmente, Platanus es un taxón que domina la primavera.

Los máximos diarios fueron variables entre curvas. En Poaceae sólo alcanzó el 6\% del total anual, mientras que en Cedrus fue del $23 \%$.

El PP y PPP de Platanus coinciden con lo encontrado por Páez (2008) para la ciudad de
Diamante. El inicio de ambos períodos en los taxones restantes presentó variaciones dependiendo de cada caso. Las diferencias entre el inicio del PP y el inicio del PPP fueron importantes (cercanas a un mes) para Cedrus y Ambrosia, mientras que para Platanus y Artemisia fueron escasos días. Esto puede deberse a un aporte de fuentes regionales de zonas con diferencias microclimáticas, y que aportan polen a la atmósfera. También puede deberse a diferencias en la maduración de los distintos ejemplares dispuestos en la ciudad. Sería importante continuar con los muestreos y correlacionar los datos polínicos con datos fenológicos y meteorológicos. En relación a esto, pueden ocurrir fenómenos de reflote de granos de polen, o sea, de reincorporación a la atmósfera de granos depositados; o de liberación temprana de granos no funcionales pero con capacidad alergizante (Latorre \& Bianchi, 1998; Latorre, 1999a).

\section{Calendario polínico}

Como refleja el calendario, la mayoría de los tipos polínicos presentaron un PP muy bien definido. No obstante, en algunos como Poaceae o Myrtaceae, la presencia en la atmósfera fue extensa, generalmente más de tres meses e incluyó polen de varias especies con distinta época de floración pero que tienen un grano de polen similar e indiferenciable bajo microscopía óptica $\mathrm{y}$ en las condiciones de estas muestras (fijos y con contenidos celular). Por el contrario, taxones como Salix, Populus, Moraceae (Morus), Platanus, Ambrosia y Artemisia, representados en vegetación por una o dos especies, se detectaron durante un periodo de tiempo muy corto.

Si bien los taxones anteriormente citados pueden considerarse como los de mayor relevancia, tanto por su elevada presencia como por la alergenicidad de muchos de ellos, no hay que descartar la importancia del resto de los taxones acompañantes. Dentro de polen arbóreo, los tipos Fraxinus, Populus y Ulmus son los principales durante los meses invernales y en los inicios de la primavera.

Los resultados obtenidos aquí, no muestran diferencias importantes en cuanto a composición de los más relevantes respecto de los de Latorre \& Perez (1997) y Bianchi (1994) obtenidos a partir de datos colectados veinte años atrás en la ciudad de Mar del Plata. Sin embargo, y teniendo en 


\section{L. Abud Sierra y F. Latorre - Polen alergénico en Mar del Plata}

cuenta los cambios en el clima y los efectos de éste sobre el crecimiento y la floración de las plantas, los estudios aerobiológicos y la construcción de los calendarios polínicos que incluya más años de muestreo son de vital importancia para obtener información detallada de las variaciones en la concentración atmosférica para identificar los PPP de aquellas plantas capaces de desencadenar enfermedades alérgicas.

\section{Estaciones de polen alergénico}

Se establecieron cinco estaciones de polen alergénico para la ciudad de Mar del Plata, que difieren de las estaciones calendario. Tres estaciones polínicas están dominadas por especies alergénicas arbóreas: una en agosto con predominio de Cupressaceae, otra en abril con Cedrus como principal componente, y otra formada por setiembre y octubre con predominio de polen de Acer y Platanus. Una cuarta estación está dominada por especies alergénicas herbáceas e incluye a noviembre y diciembre; caracterizada por la abundante presencia de Poaceae. La última estación incluye a los demás meses del año y es más heterogénea; dominan tanto especies no arbóreas como Ambrosia, Artemisia y Urticaceae, y especies arbóreas. La importancia de estas estaciones establecidas en base a la alergenicidad del polen presente en el aire, radica en su utilidad para que los médicos alergistas puedan desarrollar técnicas más eficaces en el tratamiento y prevención de los episodios de alergia en la población atópica. En el área mediterránea de España, se identifican tres estaciones polínicas según sus alérgenos predominantes: polinosis de invierno, con artemisia y ciprés, de primaveraverano con plátano, olivo, gramíneas y ortigas, y de verano-otoño con quenopodiáceas (Carbonell Martínez, 2013). En base a lo anterior, podríamos definir en Mar del Plata: polinosis de primavera en setiembre y octubre con plátano y arce, polinosis de fines primavera-principios de verano en noviembre y diciembre con gramíneas, polinosis de fines verano-principios otoño en febrero y marzo con ambrosia, artemisia y ortigas, polinosis de mediados otoño en abril con cedro, polinosis de fines invierno en agosto con ciprés. Este análisis representa una primera aproximación hacia el establecimiento de estaciones polínicas basadas en la presencia de las especies dominantes alergénicas en el aire de la ciudad.
Debe destacarse además, que no se han registrado días sin polen en el aire, ni aún durante el invierno (estación en la cual se registra un mínimo de polen en el aire); en todo momento se encuentra polen en suspensión con capacidad potencial para causar síntomas alergénicos en las personas sensibles.

\section{Conclusiones}

A partir del estudio del espectro polínico anual de la ciudad de Mar del Plata, se concluye lo siguiente:

Se registraron 50 tipos polínicos diferentes, principalmente de especies arbóreas de uso ornamental como son: Cupressaceae, Cedrus, Ulmus, Fraxinus, Platanus.

La concentración mensual total aumenta desde invierno hasta la primavera y principios de verano, y luego desciende; agosto es el mes con mayor concentración total de polen y junio el de menor valor

Los tipos polínicos de las especies arbóreas dominan el espectro urbano durante la mayor parte del año.

La mayor concentración de polen no arbóreo se registró durante el mes de diciembre, siendo Poaceae el taxón dominante.

El $95 \%$ del polen presente en la atmósfera de la ciudad de Mar del Plata corresponde a tipos polínicos considerados alergénicos o potencialmente alergénicos.

El gran aporte de Cupressaceae y Cedrus podría potenciar su alergenicidad. Los síntomas alérgicos provocados por estos dos tipos polínicos se relacionan con el tiempo de permanencia en el aire y su abundancia, junto con otros factores de riesgo (Caimmi, 2012 y Lorenzoni-Chiesura, 2000).

El calendario polínico confeccionado permitió determinar el comportamiento de los tipos polínicos más abundantes y alergénicos en la atmósfera de la ciudad, y actualizar la información aerobiológica luego de 20 años sin información.

La determinación de estaciones de polen alergénico en lugar de estaciones calendario, brinda información más valiosa a los médicos alergistas para la prevención de las enfermedades respiratorias.

Se continúa con el monitoreo atmosférico de la atmósfera de la ciudad con el objetivo de obtener un calendario polínico representativo también de la 
variación interanual que ocasiona la meteorología y aquellas especies que alternan años de elevada producción polínica con años de baja polinización. Actualmente, la información aerobiológica de Mar del Plata se reporta diariamente mediante la página web de la Asociación Argentina de Alergia e Inmunología Clínica (Latorre \& Abud Sierra, 2017). Estos sistemas de información pueden ser utilizados por la población de riesgo y los profesionales sanitarios en la aplicación de medidas preventivas oportunas en relación a las alergias respiratorias en nuestra ciudad.

Se debería continuar poniendo énfasis en el seguimiento de las especies que promueven polinosis, y completar con estudios epidemiológicos de casos para conocer la incidencia directa en la población de la ciudad de Mar del Plata. Estos resultados pueden ayudar en la toma de medidas orientativas respecto del planeamiento y diseño del arbolado urbano, fomentando la utilización de aquellas especies que no sean potenciales promotores de enfermedades y reducir la posibilidad de los casos de polinosis.

\section{Contribución de los autores}

Los datos de este trabajo fueron parte de la tesis de licenciatura realizada por MLAS bajo la dirección de FL. Este trabajo fue realizado en partes iguales por las dos autoras.

\section{Agradecimientos}

Las autoras agradecen a la Universidad Nacional de Mar del Plata (subsidio EXA 835/17 y EXA 940/19 otorgado a FL), a la Asociación Argentina de Alergia e Inmunología Clínica (Servicio Tecnológico de Alto Nivel STAN CONICET de FL a través del Instituto de Investigaciones Marinas y Costeras IIMyC, CONICET-FCEyN UNMDP), y al Servicio Meteorológico Nacional por los datos meteorológicos brindados.

\section{Bibliografía}

ABUD SIERRA, M. L., F. LATORRE \& F. BAILLEAU. 2015. Monitoreo de polen aéreo y de los trastornos alérgicos en Mar del Plata a lo largo del año. Actas XXXVIII Congreso Anual AAAeIC. 13- 15 de Agosto de 2015, Capital Federal, Buenos Aires, Argentina.

ARDUSSO, L, J. A. ROSSO, T. HERERO, M. ISORNA, E. JARES, M. I. GRILLO, M. KOHAN, M. POUSIFF, L. BARRIONUEVO \& G. RAMÓN. 2010. Prevalencia de sensibilidad cutánea a 12 especies de pólenes de árboles aerotransportados en pacientes con asma y/o rinitis residentes en el área de la Ciudad Autónoma de Buenos Aires. Archivos de Alergia e Inmunología Clínica 4: 54-60.

BARONI, M. V., J. S. ALVAREZ, D. A. WUNDERLIN \& G. A. CHIABRANDO. 2008. Analysis of IgE binding proteins of Celtis tala pollen. Food and agricultural immunology 19: 187-194. https://doi.org/10.1080/09540100802190765

BIANCHI, M. M. 1992. Calendario polínico de la ciudad de Mar del Plata (Agosto 1987 - Agosto 1989). Arch. Arg. Alerg. Inmunol. Clin. 23: 73-86.

BIANCHI M. M. 1994. El muestreo aerobiológico en Mar del Plata. Aportes de una nueva metodología al análisis de polen. Su aplicación en el diagnóstico de la polinosis. Academia Nacional de Ciencias, Monografia 10. Ediciones Sigma SRL, Buenos Aires.

BOUSQUET, J., J. KNANI, A. HEJJAOUI, P. FERNANDO, P. COURN \& H. DHIVERT. 1993. Heterogeneity of atopy. I. Clinical and Inmunologic caractheristics of patients allergic cippres pollen. Allergy 48: 183-188. https://doi.org/10.1111/j.1398-9995.1993.tb00711.x

CABRERA, A. L. 1958. Fitogeografia. En: DE APARICIO F. \& DIFRIERI H.A. (eds.) La Argentina. Suma de geografia. Tomo 3. Peuser. Buenos Aires. Argentina.

CABRERA, A.L. \& E. ZARDINI. 1978. Manual de la flora de los alrededores de Buenos Aires. ACME, Buenos Aires. Argentina.

CAIMMI, D., R. RASCHETTI, P. PONS, H. DHIVERTDONNADIEU, P. J. BOUSQUET, J. BOUSQUET \& P. DEMOLY. 2012. Epidemiology of Cypress pollen allergy in Montpellier. Journal Investigation Allergollogy Clinical Immunology 22: 280-285.

CARBONELL MARTÍNEZ, A. 2013. Etiología y fisiopatología de la polinosis. En: Pólenes alergénicos y polinosis en la Región de Murcia.

CARIÑANOS, P., P. ALCÁZAR, C. GALÁN \& E. DOMÍNGUEZ. 2002. Privet pollen (Ligustrum $s p$.) as potential cause of pollinosis in the city of Cordoba, south-west Spain. Allergy 57: 92-97. https://doi.org/10.1034/j.1398-9995.2002.103261.x 


\section{L. Abud Sierra y F. Latorre - Polen alergénico en Mar del Plata}

CASTRO ALFAGEME, S. D. 2015. Estudio de alérgenos de polen en la atmósfera de Valladolid. Tesis doctoral. Universidad de León. España.

CID MARTINEZ, M. A, R. L. FÓCIL MONTERRUBIO \& J. E ROSIQUE GIL. 2013. Inventario polínico de una zona suburbana del municipio de Centro, Tabasco. Revista de divulgación académica de Ciencias Biológicas 19: 19-21.

D'AMATO, G, L. CECCHI, S. BONINI, C. NUNES, I. ANNESI-MAESANO \& H. BEHRENDT. 2007. Allergenic pollen and pollen allergy in Europe. Allergy 62:976-90. https://doi.org/10.1111/j.1398-9995.2007.01393.x

DE LA PLAZA AGUADO, C. 2018. Estudio de alérgenos a través de métodos inmunológicos. Tesis Doctoral Universidad Complutense. Pp. 20.

EDMONDS, R. L. \& W. S. BENNINGHOFF. 1973. Aerobiology and Its Modern Applications: A discipline of investigations of aerial transport of biological materials important to human health and welfare. US Component of the International Biological Program, Aero. Program. 1-18.

ERDTMAN, G. 1954. An introduction to Pollen Analysis. Chronica Botanica Company Press, Waltham, Massachusetts.

ERDTMAN, G. 1971.Pollen morphology and plant taxonomy. Angiosperms. Hafner Publishing Co. New York.

FERNÁNDEZ-LLAMAZARES I., J. BELMONTE, M. BOADA, S. FRAIXEDAS. 2014. Airborne pollen records and their potential applications to the conservation of biodiversity. Aerobiologia 30:111-122. https://doi. org/10.1007/s10453-013-9320-4

FERREIRA, F., G. GADERMAIER \& M. WALLNER. 2014. Alérgenos de polen de árboles. Capítulo 3f. In: AKDIS C.A. \& I. AGACHE (eds.), Global atlas of allergy, pp:18-21. European Academy of Allergy and Clinical Immunology.

GALÁN, C., A. ARIATTI, M. BONINI, B. CLOT, B. CROUZY, A. DAHL, D. FERNANDEZ-GONZALEZ, G. FRENGUELLI, R. GEHRING, S. ISSARD, E. LEVETIN, D. W. LI, P. MANDRIOLI, C. A. ROGERS, M. THIBAUDON, I. SAULIENE, C. SKJOTH, M. SMITH \& M. SOFIEV. 2017. Recommended terminology for aerobiological studies. Aerobiologia 33: 293-295.

https://doi.org/10.1007/s10453-017-9496-0

GARCÍA, M. E. 2010. Aeropalinología de la ciudad de Yerba Buena, provincia de Tucumán (Argentina). Acta Botánica Malacitana 35: 115-131. https://doi.org/10.24310/abm.v35i0.2870
GARCIA-GONZALEZ, J. J., B. BARTOLOMEZAVALA, M. M. TRIGO-PÉREZ, J. M. BARCELOMUÑOZ, S. FERNANDEZ-MELENDEZ, M. A. NEGRO-CARRASCO \& R. PALACIOS-PELAEZ. 1999. Pollinosis to Ricinus communis (castor bean): an aerobiological, clinical and immunochemical study. Clinical and experimental allergy 29: 12651275 .

https://doi.org/10.1046/j.1365-2222.1999.00601.x

GARCÍA MOZZO, M. H. 2002. Optimización de modelos predictivos a corto y largo plazo de polen tipo Quercus en distintas zonas climáticas españolas. Tesis doctoral. Córdoba, España.

GINER, M. M. 1999. Patrones de variación polínica en la atmósfera de Murcia: implicaciones alergológicas, prevención y diagnóstico. Tesis doctoral. Universidad de Murcia, España.

HEUSSER, C. J. 1971. Pollen and spores of Chile. Univ. Arizona Press, Tucson. USA. https://doi.org/10.2307/1218275

HIRST J. M. 1952. An automatic volumetric spore trap. Ann. Appl. Biol. 39: 257-265. https://doi.org/10.1111/j.1744-7348.1952.tb00904.x

HUECAS GAYO, S. 2001. Ole e 9, a major olive pollen allergen is a 1,3-ß-glucanase. Isolation, characterization, amino acid sequence, and tissue specificity. J. Biol. Chem. 276: 27959-27966. https://doi.org/10.1074/jbc.M103041200

HUERTAS AMORÓS, A. J. 2013. Polinosis: concepto e introducción. En: MIRALLES LÓPEZ, J. C. \& J. M. NEGRO ÁlVAREZ, J.M. (eds.), Pólenes Alergénicos y Polinosis en la Región de Murcia, pp. 23-29. AlergoMurcia. Asociación de Alergología e Inmunología Clínica de la Región de Murcia. ISBN 13: 978-84-695-7172-9.

HUSSAIN I. \& J. SMITH. 2003. Evidence for the transmissibility of atopy. Chest 124: 1968-1974. https://doi.org/10.1378/chest.124.5.1968

IBARROLA, I., M. C. ARILLA, A. MARTÍNEZ \& J. A. ASTURIAS. 2004. Identification of a polygalacturonase as a major allergen (Pla a 2) from Platanus acerifolia pollen. J. Allergy Clin. Immunol. 113: 1185-1191. https://doi.org/10.1016/j.jaci.2004.02.031

JATO M. V., I. IGLESIAS, M. J. AIRA. 2000. Atlas de polen alergógeno. Ed. Xunta de Galicia, Galicia.

KÄPYLÄ, M. \& A. PENTTINEN. 1981. An evaluation of microscopical counting methods of the tape in Hirst- pollen and spores. Grana 20: 131-141. https://doi.org/10.1080/00173138109427653 
KOCHIBE, N. 1997. Allergic substances of Ginkgo biloba. Ginkgo biloba A Global Treasure. Springer, Tokyo. https://doi.org/10.1007/978-4-431-68416-9_23

LATORRE, F. 1993. Relación entre la concentración de polen en la atmósfera y la vegetación arbórea de la ciudad de Mar del Plata. Tesis de Licenciatura. Universidad Nacional de Mar del Plata, Argentina.

LATORRE, F. 1997. Comparison between phenological and aerobiological patterns of some arboreal species of Mar del Plata (Argentina). Aerobiologia 13: 4959. https://doi.org/10.1007/BF02694791

LATORRE, F. 1999a. El polen atmosférico como indicador de la vegetación y de su fenología floral. Tesis doctoral UBA, Argentina.

LATORRE, F. 1999b. Differences between airborne pollen and flowering phenology of urban trees with reference to production, dispersal and interannual climate. Aerobiologia 15: 131-141.

LATORRE, F. \& M.M., BIANCHI. 1997. Relación entre la concentración polínica atmosférica y la vegetación arbórea de Mar del Plata (Argentina). Polen 8: 43-59.

LATORRE, F, \& C.F., PEREZ. 1997. One year of airborne pollen sampling in Mardel Plata(Argentina). Grana 36: 49-53. https://doi.org/10.1080/00173139709362590

LATORRE, F. \& M. M., BIANCHI 1998. Relationships between flowering devolopment of Ulmus pumilia and Fraxinus excelsior and their airbone pollen. Grana 37: 233-238.

https://doi.org/10.1080/00173139809362672

LATORRE, F. \& M.A. CACCAVARI. 2009. Airborne pollen patterns in Mar del Plata atmosphere (Argentina) and its relationship with meteorological conditions. Aerobiologia 25: 297- 312. https://doi.org/10.1007/s10453-009-9134-6

LATORRE, F. \& M.A. CACCAVARI. 2010. Pollen diversity in the air of Diamante (Entre Ríos, Argentina). Scientia Interfluvius 1: 07-17.

LATORRE, F. \& M. L. ABUD SIERRA 2017. Principales tipos polínicos en el aire de Mar del Plata (setiembre 2013-marzo 2017). XL Congreso anual AAAeIC. 10-12 agosto. Buenos Aires. Archivos de Alergia e Inmunología Clínica 48: 53-54.

LATORRE, F., E. J. ROMERO \& M. V. MANCINI. 2001. Representatividad de la vegetación en el espectro de polen atmosférico de la ciudad de Mar del Plata (Argentina). Asociación Paleontológica Argentina. Ameghiniana 8: 119-124.

LATORRE, F., E. J. ROMERO \& M. V. MANCINI. 2008. Comparative study of different methods for capturing airborne pollen, and effects of vegetation and meteorological variables. Aerobiologia 24: $107-$ 120. https://doi.org/10.1007/s10453-008-9090-6

LATORRE F, M. L. ABUD SIERRA, F. BAILLEAU. 2014. Diversidad y estacionalidad del polen de Mar del Plata durante primavera-verano y su relación con síntomas alérgicos. Actas del XXXVII Congreso Anual AAAeIC. 14-16 de Agosto de 2014, Capital Federal, Buenos Aires, Argentina.

LORENZONI-CHIESURA F., M. GIORATO \& G. MARCER. 2000. Allergy to pollen of urban cultivated plants. Aerobiologia 16: 313-316. https://doi.org/10.1023/A:1007652602113

MARCHUET, M. J. 2016. Alergia a cupresáceas en la población del Vallés Occidental. Tesis Doctoral. Universitat Autònoma de Barcelona. Spain.

MARCÓ, L. N., E. P. FOTI, P. I. COSTA, R. R. AZARIO, D. BUENO, M. N., M. CHICHI, A. COSTA, D. LIMA, M. PIROVANI \& F. LATORRE. 2014. Aportes a la Epidemiología del asma infantil. Ciencia, Docencia y Tecnología Suplemento 4: 182-205.

MARKGRAF, V. \& H. L. D’ANTONI. 1978. Pollen flora of Argentina. Modern spore and pollen types of Pteridophyta, Gymnospermae and Angiospermae. Univ. Arizona Press, Tucson.

MATHOV. E. 1972 Curso completo de alergia e inmunología para clínicos y pediatras. Tomo 1 . Editorial Publicaciones médicas argentinas.

MILLARES LÓPEZ, J. C. 2015. Polinosis, una morbilidad en aumento: la importancia de los recuentos aerobiológicos en la práctica clínica. Revista de Salud Ambiental 15: 42-45.

MOORE, P. D. \& J. A. WEBB. 1978. An Illustrated Guide to Pollen Analysis. Hodder and Stoughton. London - Sydney Auckland - Toronto.

MOORE, P. D., J. A. WEBB \& M. E. COLLINS. 1991. Pollen Analysis. Blackwell Sci. Publ., Oxford London - Edinburgh - Boston - Melburn.

MURRAY, G. 2006. Un estudio del polen aerotransportado en Bahía Blanca y su región. Tesis doctoral.

MURRAY, M. G., M. I. SONAGLIONI \& C. B. VILLAMIL. 2002. Annual variation of airborne pollen in the city of Bahía Blanca, Argentina. Grana 41: 183-189.

https://doi.org/10.1080/001731302321042632

NILSSON, S. \& S. PERSSON. 1981. Tree pollen spectra in the Stockholm region (Sweden), 1973-1980. Grana 20: 179-182. https://doi.org/10.1080/00173138109427661 


\section{L. Abud Sierra y F. Latorre - Polen alergénico en Mar del Plata}

NITIU, D. S. 2006. Aeropalynologic análisis of La Plata city (Argentina) during a 3-years period. Aerobiologia 22: 79-87. https://doi.org/10.1007/s10453-005-9009-4

NITIU, D. S. 2009. Estudio del polen atmosférico y su relación con la vegetación local. La Plata, Argentina. Act. Bot. Mal. 34: 1-11. https://doi.org/10.24310/abm.v34i0.6938

NITIU, D.S., A. C. MALLO, \& E. J. ROMERO, 2003. Quantitative aeropalynology in the atmosphere of Buenos Aires city, Argentina. Aerobiologia 19: 1-1. https://doi.org/10.1023/A:1022641015237

PÁEZ, C. 2008. Estudio del polen de la atmósfera de Diamante (Entre Ríos, Argentina): Patrón de distribución intradiaria, representatividad de la vegetación urbana y nativa, y relación con la polinosis. Tesis de Licenciatura. FCyT. Universidad Autónoma de Entre Ríos. Entre Ríos. Argentina.

PEREZ, C. F. 2000. Caracterización de la nube polínica y determinantes meteorológicos de la dispersión del sistema urbano-rural de Mar del Plata. Tesis doctoral. FCEyN. Universidad Nacional de Mar del Plata.

PIRE S.M., L.M. ANZÓTEGUI \& G.A. CUADRADO. 1998. Flora polínica del nordeste argentino Vol. I (EUDENE UNNE) Universidad Nacional del Nordeste, Argentina.

PIRE S.M., L.M. ANZÓTEGUI \& G. A. CUADRADO. 2001. Flora polinica del nordeste argentino Vol. II (EUDENE UNNE) Universidad Nacional del Nordeste, Argentina.

PLA DALMAU J. M. 1961. Polen. Estructura $y$ características de los granos de polen. Preciciones morfológicas sobre el polen de especies recolectadas en el N. Ede España. Polinización y aeropalinología. Ed. Tall. Graf. DCP. Gerona, España.

PUIGGRÒS CASAS, A. 2012. Prevalencia de sensibilización a los pólenes de árboles plantados en la ciudad de Barcelona. Tesis doctoral. Universitàt Autònoma de Barcelona.

ROGERS, C \& M. MUILENBERG. 2001. Comprehensive Guidelines for the operation of Hirst-Type suction bioaerosol samplers. Pan-American Aerobiology Association, Standarized Protocols. Disponible en http://www. paaa. org/StandardizedProtocols. Pdf
ROUBIK, D.W. \& J. E. MORENO. 1991. Pollen and Spores of Barro Colorado Island. Missouri Botanical Garden 36: 270 pp.

SALMUD, N. \& L. M. SALMUD. 2016. Mitos y realidades sobre el asma y la alergia. Disponible en https://www.fundaler.org.ar/publicaciones/ otros-folletos-de-fundaler/mitos-y-realidades sobre-el asma-y-la-alergia/ [Acceso: 11 enero 2019].

SIMÓN, B.E., F. LATORRE \& C. ROTUNDO. 2018. Study of the reproductive phenology of Araucaria angustifolia in two environments of Argentina: Its application to the management of a species at risk. Global Ecology and Conservation 16. https://doi.org/10.1016/j.gecco.2018.e00483

TEJERA. L., \& A. BERI 2005. First volumetric airborne pollen sampling in Montevideo City, Uruguay. Aerobiología 21: 33-41. https://doi.org/10.1007/s10453-004-5876-3

TERÁN, L. M., M. HASELBARTH, \& D. QUIROZ, 2009. Alergia, pólenes y medio ambiente. Gac Méd Méx 145: 215-22.

TORRES, G. R., \& F. F. FLORES. 2013. Reporte de polen atmosférico durante la estación primaveral de 2007 en la ciudad de San Salvador de Jujuy, Argentina. Revista Mus. Argent. Ci. Nat. "Bernardino Rivadavia." 15: 175-182. https://doi.org/10.22179/REVMACN.15.179

VALERO, S. A. L \& G. A. CAHIA. 2002. Polinosis. Polen y alergias. MRA Ediciones, S.L.

VAN REE, R., W. A. VAN LEEUWEN \& R. C. ALBERSE. 1998. How far can we simplify in vitro diagnostics for grass pollen allergy? A study with 17 whole pollen extracts and purified natural and recombiant major allergens. Journal or allergy and clinical immunology 102: 184-190. https://doi.org/10.1016/S0091-6749(98)70084-3

WODEHOUSE, R. 1965. Pollen grains. Their structure, identification and significance in Science and Medicine. Hafner, New York, fascimile of 1935 edition. https://doi.org/10.2307/2419987

UNIÓN INTERNACIONAL DE SOCIEDADES DE INMUNOLOGÍA. 2019. Disponible en: http://www. allergen.org/search.php [Acceso: 10/8/2019]. 
